\title{
0620 CIRCUMSTANCES OF SLIPS TRIPS AND FALLS AMONG HOSPITAL WORKERS
}

T K Courtney*, D A Lombardi, G S Sorock, H M Wellman, S Verma, M J Brennan, J Collins, J Bell, WR Chang, R Grnqvist, L Wolf, E DeMaster, M Matz Correspondence: Center for Injury Epidemiology, Liberty Mutual Research Institute for Safety, 71 Frankland Road Hopkinton, MA 01748, USA

\subsection{6/ip.2010.029215.620}

A case follow-back study was conducted to better describe the circumstances experienced by hospital workers who had slipped, tripped and/or fallen (STF). One hundred 53 healthcare workers, who reported a STF to the occupational health department in one of four private and three public US hospitals, were recruited and interviewed using a structured telephone questionnaire. Participants were predominantly female (86\%) with a mean age (range) of 46 (19-67). One hundred and thirty-six workers (89\%) fell: 55\% after slipping, 32\% after tripping. Liquid contaminants (eg, water, cleaning solutions) were involved in $35 \%$ of events. Fifty-eight percent of STF occurred at transitional areas, of these: $34 \%$ were dry/wet transitions, $18 \%$ one floor type to another (eg, carpet to tile), $14 \%$ uneven surfaces. Forty-five percent of workers fell forward, 29\% fell to the side and $23 \%$ fell backward. While the wrists/hands, knees and buttocks were most often the points of impact, the knees, back, ankles/feet were most frequently injured. For injured workers (93\%), strains and sprains (28\%), contusions $(28 \%)$ and non-specific pain and soreness (24\%) were typical. Fiftynine percent of workers who experienced STF were involved in direct patient care occupations (eg, nursing, therapy). Forty- 


\section{IP Safety 2010 abstracts}

two percent of worker STF occurred in public areas inside the facility, $25 \%$ in public areas outside the facility, and $18 \%$ in direct patient care areas (including intensive care, operating rooms and patient rooms). The applications to worker safety and health are discussed along with the potential implications for visitor and patient safety. 